\title{
Compensation for air voids in photoacoustic computed tomography image reconstruction
}

Thomas P. Matthews, Lei Li, Lihong V. Wang, Mark A. Anastasio

Thomas P. Matthews, Lei Li, Lihong V. Wang, Mark A. Anastasio, "Compensation for air voids in photoacoustic computed tomography image reconstruction," Proc. SPIE 9708, Photons Plus Ultrasound: Imaging and Sensing 2016, 970841 (15 March 2016); doi: 10.1117/12.2213307

SPIE. Event: SPIE BiOS, 2016, San Francisco, California, United States 


\title{
Compensation for air voids in photoacoustic computed tomography image reconstruction
}

\author{
Thomas P. Matthews ${ }^{\mathrm{a}}$, Lei Li ${ }^{\mathrm{a}}$, Lihong V. Wang ${ }^{\mathrm{a}}$, and Mark A. Anastasio ${ }^{\mathrm{a}}$ \\ aDepartment of Biomedical Engineering, School of Engineering and Applied Science, \\ Washington University in St. Louis, St. Louis, MO 63130
}

\begin{abstract}
Most image reconstruction methods in photoacoustic computed tomography (PACT) assume that the acoustic properties of the object and the surrounding medium are homogeneous. This can lead to strong artifacts in the reconstructed images when there are significant variations in sound speed or density. Air voids represent a particular challenge due to the severity of the differences between the acoustic properties of air and water. In whole-body small animal imaging, the presence of air voids in the lungs, stomach, and gastrointestinal system can limit image quality over large regions of the object. Iterative reconstruction methods based on the photoacoustic wave equation can account for these acoustic variations, leading to improved resolution, improved contrast, and a reduction in the number of imaging artifacts. However, the strong acoustic heterogeneities can lead to instability or errors in the numerical wave solver. Here, the impact of air voids on PACT image reconstruction is investigated, and procedures for their compensation are proposed. The contributions of sound speed and density variations to the numerical stability of the wave solver are considered, and a novel approach for mitigating the impact of air voids while reducing the computational burden of image reconstruction is identified. These results are verified by application to an experimental phantom.
\end{abstract}

Keywords: photoacoustic computed tomography, image reconstruction, acoustic heterogeneity

\section{INTRODUCTION}

Photoacoustic computed tomography (PACT) is a hybrid imaging modality that combines optical image contrast with the high resolution of acoustic detection. ${ }^{1,2}$ It has a wide variety of potential applications, ranging from pre-clinical small animal imaging to clinical human measurements. ${ }^{2}$ In PACT, a short optical pulse is used to illuminate an object of interest. The light is then absorbed, resulting in the generation of an acoustic wave via the photoacoustic effect. The pressure wave is measured by a collection of ultrasound transducers surrounding the object. The image reconstruction problem is to estimate the absorbed optical energy density, or equivalently the initial pressure distribution, from the pressure measurements collected at the transducers.

Most PACT image reconstruction methods are based on simplistic models that assume that the medium is acoustically homogeneous. ${ }^{3}$ This assumption is often violated in practice. For example, in small animal imaging, air voids exist in the lungs, stomach, and gastrointestinal system. Ignoring these sources of acoustic heterogeneity can lead to artifacts in the reconstructed images. ${ }^{4-6}$ Methods based on the acoustic wave equation are able to overcome this limitation by accounting for variations in the sound speed and density. However, strong acoustic heterogeneities can lead to instability or phase errors in numerical wave solvers. Given the large differences between the acoustic properties of water (or tissue) and air, accounting for air voids represents a significant challenge.

Here, we consider the impact of air voids on PACT image reconstruction and give procedures for their compensation. The contributions of sound speed and density variations to the numerical stability of the wave solver are considered, and a novel approach for mitigating the impact of air voids while reducing the computational burden of image reconstruction is identified.

Further author information: (Send correspondence to Mark A. Anastasio, Lihong V. Wang)

Mark A. Anastasio: E-mail: anastasio@wustl.edu,

Lihong V. Wang: Email: lhwang@seas.wustl.edu

Photons Plus Ultrasound: Imaging and Sensing 2016, edited by Alexander A. Oraevsky, Lihong V. Wang Proc. of SPIE Vol. 9708, 970841 - @ 2016 SPIE · CCC code: 1605-7422/16/\$18 - doi: 10.1117/12.2213307 


\section{THEORY}

In this work, we employ the first-order k-space pseudospectral wave solver. ${ }^{7,8}$ Ignoring attenuation and dispersion and assuming the wave propagation is linear, this approach solves the pair of coupled first-order differential equations

$$
\begin{gathered}
\rho(\mathbf{r}) \frac{\partial \mathbf{u}(\mathbf{r}, t)}{\partial t}=-\nabla p(\mathbf{r}, t) \\
\frac{1}{\rho(\mathbf{r}) c(\mathbf{r})^{2}} \frac{\partial p(\mathbf{r}, t)}{\partial t}=-\nabla \cdot \mathbf{u}(\mathbf{r}, t),
\end{gathered}
$$

where $\rho(\mathbf{r})$ is the density, $c(\mathbf{r})$ is the sound speed, $\mathbf{u}(\mathbf{r}, t)$ is the particle velocity, and $p(\mathbf{r}, t)$ is the pressure. When these coupled differential equations are solved numerically, it is necessary to introduce discrete representations of the above variables. Given that the first-order k-space method involves frequent computation of the Fourier transform, it is natural to employ a pixel-basis. For simplicity, we assume here that the pixels are square. Ignoring the density variations, the wave solver is numerically stable when ${ }^{7,9}$

$$
\sin (\pi \mathrm{CFL} / 2) \leq \frac{c_{r e f}}{c_{\max }},
$$

where CFL is the Courant-Friedrichs-Lewy number, defined as CFL $\equiv c_{r e f} \Delta t / \Delta x, \Delta t$ is the time step, $\Delta x$ is the pixel size along one dimension, $c_{\text {ref }}$ is the reference sound speed used in the k-space operator, and $c_{\max }$ is the largest sound speed value over the simulation grid. In practice, it is often necessary to satisfy even stricter criteria when the density is heterogeneous and to avoid significant phase errors. For example, we found that $\Delta x \leq \lambda_{\min } / 3$ resulted in better numerical accuracy for strongly acoustically heterogeneous media. As a result, the reference sound speed, the pixel size, and the time step were chosen based on the following criteria:

$$
\begin{aligned}
c_{\text {ref }} & =c_{\text {max }} \\
\Delta x & \leq \frac{\lambda_{\min }}{3}=\frac{c_{\min }}{3 f_{\max }} \\
\Delta t & \leq \frac{\eta \Delta x}{c_{\text {ref }}} \quad(\mathrm{CFL} \leq \eta),
\end{aligned}
$$

where $\lambda_{\min }$ is the smallest wavelength that must be simulated, $f_{\max }$ is the maximum temporal frequency, $c_{\min }$ is the smallest sound speed value over the simulation grid, and $0<\eta<1$ is a constant. When air voids are present, these criteria can result in very strict conditions for $\Delta x$ and $\Delta t$. For example, consider the case where $f_{\max }=5 \mathrm{MHz}$. Then, using the stricter criteria, $\Delta x \leq 22 \mu \mathrm{m}$ and, for $\eta=0.5, \Delta t \leq 7.4 \mathrm{~ns}$ (equivalent to a sampling frequency of $135 \mathrm{MHz}$ ).

Here, we propose a heuristic method for reducing the computational burden associated with the numerical wave solver. Due to the large differences in the acoustic properties of air and tissue (or water), most of the energy of an incoming pressure wave is reflected at the air-tissue interface. This holds true even if only density variations are considered. For example, consider the energy reflected at a planar interface for a normally incident wave $^{10}$

$$
R_{\text {energy }}=\frac{\left(Z_{w}-Z_{a}\right)^{2}}{\left(Z_{w}+Z_{a}\right)^{2}},
$$

where $Z=\rho c$ is the acoustic impedance, $Z_{w}$ is the acoustic impedance of water, and $Z_{a}$ is the acoustic impedance of air. When both sound speed and density variations are considered, $R_{\text {energy }}=0.999$. When only density variations are considered, $R_{\text {energy }}=0.995$. By ignoring the sound speed variations, the minimum wavelength $\lambda_{\min }$ is greatly increased, allowing use of much larger step sizes. This increased step size further permits use of a large time step, further reducing the computational burden of the numerical wave solver. This strategy could be particularly useful for 3D image reconstruction problems, where the small pixel size necessitated by the air voids might otherwise make the problem computational infeasible. 


\section{NUMERICAL SIMULATION STUDIES}

\subsection{Methods}

Numerical phantoms for the initial pressure distribution, the sound speed, and the density were generated and are shown in Fig. 1. This phantom was chosen to mimic an experimental phantom, described in Section 4. The measurement system consisted of a circular transducer array with 512 transducers and a radius of $25 \mathrm{~mm}$.

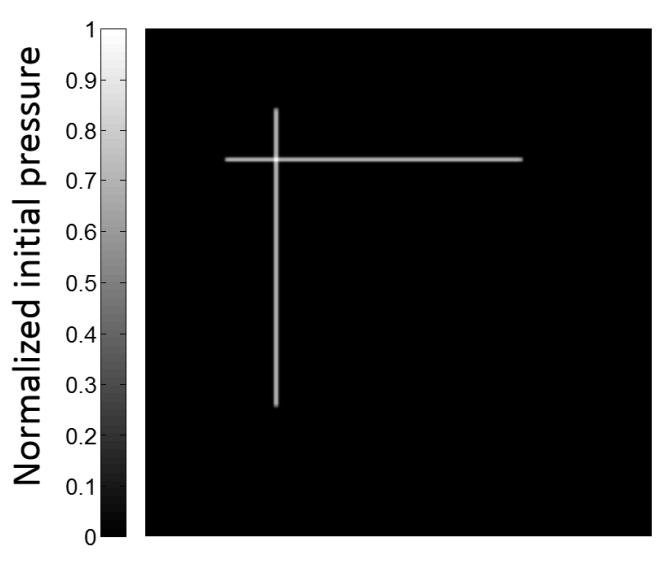

(a)

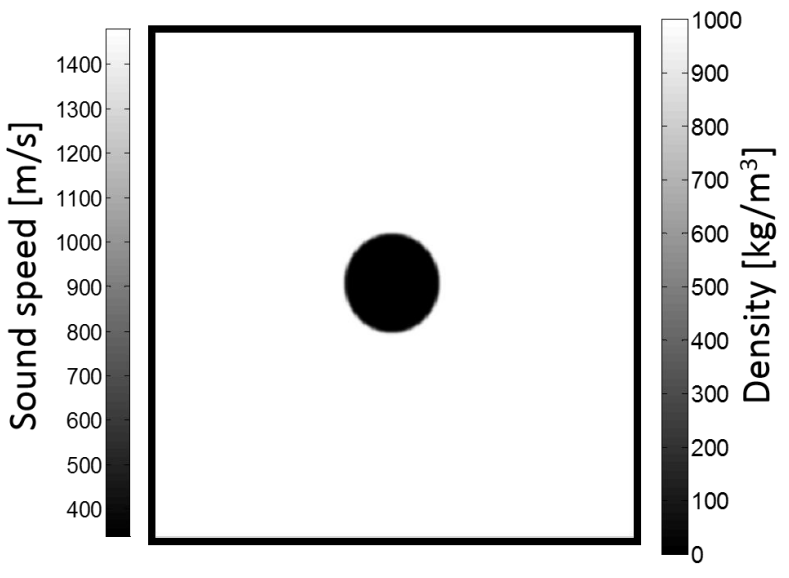

(b)

Figure 1: Numerical phantoms of (a) the normalized initial pressure distribution, (b) the sound speed and the density.

The transducers were evenly distributed about the ring. The phantoms were discretized on a 2D Cartesian grid with $2560 \times 2560$ pixels and a pixel size of $20 \mu \mathrm{m}$. The pressure data at the transducer locations was simulated using the k-Wave toolbox. ${ }^{11}$ The simulation was run for 3870 time steps with a sampling rate of $148 \mathrm{MHz}$, corresponding to about $32.25 \mu \mathrm{s}$. In this case, $\eta=0.5$. The images were reconstructed using a full-wave iterative image reconstruction method based on the MFISTA-TV optimization method. ${ }^{3,12}$ In this approach, an estimate of the absorbed optical energy density is obtained by minimizing an objective function consisting of a data fidelity term, which quantifies the agreement with the measured data, and a total-variation (TV) regularization function. The absorbed optical energy density was not estimated within the air void. For most results, the same pixel size and time step were employed for the reconstruction. When evaluating the heuristic strategy of modeling only density variations, a $1280 \times 1280$ grid was employed with a pixel size of $40 \mu \mathrm{m}$. The sampling rate was likewise decreased to $74 \mathrm{MHz}$. This resulted in the same CFL number for both pixel sizes. A regularization parameter value of $10^{-4}$ was employed in all cases.

\subsection{Results}

The impact of ignoring air voids is seen in Fig. 2. When the medium is assumed to be acoustically homogeneous, the reconstructed image possesses artifacts that are seen emanating from the object towards the source of heterogeneity (see Fig. 2a). These artifacts are absent in the reconstructed image obtained assuming the true acoustically heterogeneous medium (see Fig. 2b).

Similar errors are seen when the location of the air void is not precisely known. Images were reconstructed assuming the true size, shape, and acoustic properties of the air void, but with the center of the air void shifted from its true location. A shift, even as small as $0.2 \mathrm{~mm}$, is enough to result in highly visible artifacts (see Fig. 3). Determining the air void location through trial and error is hindered by the computational burden resulting from the strict numerical stability and accuracy criteria described in Section 2.

Here, a heuristic strategy for reducing the computational burden by assuming a constant sound speed, but a variable density is proposed. Images were reconstructed with a larger pixel size of $40 \mu m$ and a smaller sampling rate of $74 \mathrm{MHz}$ assuming both a homogeneous sound speed and the true sound speed. From the error maps in Fig. 4, it is clear that, under these conditions, assuming a constant sound speed results in a more accurate 


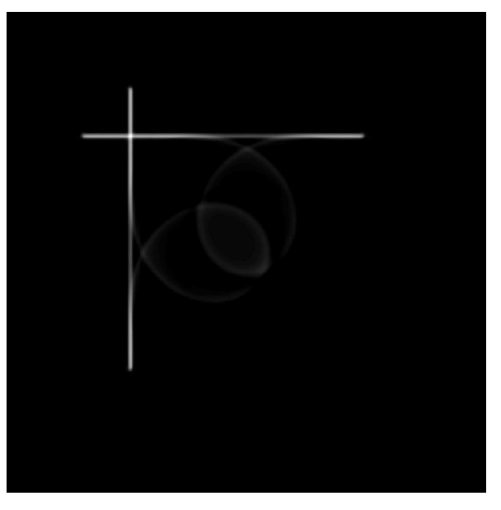

(a)

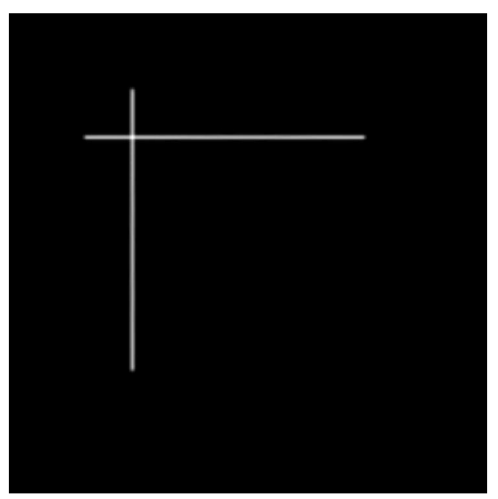

(b)

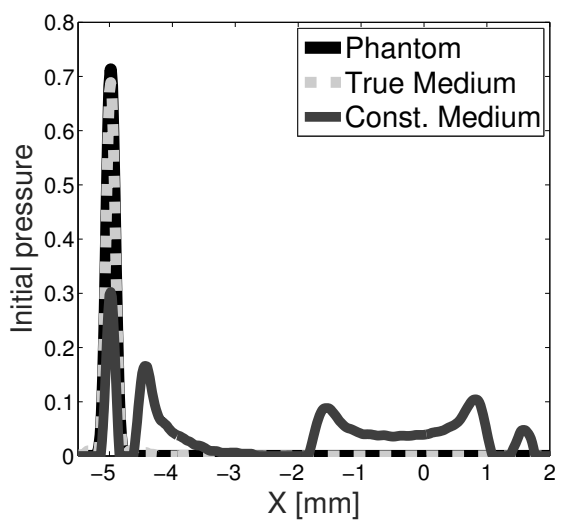

(c)

Figure 2: Images reconstructed using MFISTA assuming (a) a homogeneous acoustic medium and (b) the true acoustically heterogeneous medium. Images are shown after 40 iterations in a grayscale window of [0.0, 1.0]. (c) Profiles through the reconstructed images at $\mathrm{y}=0 \mathrm{~mm}$.

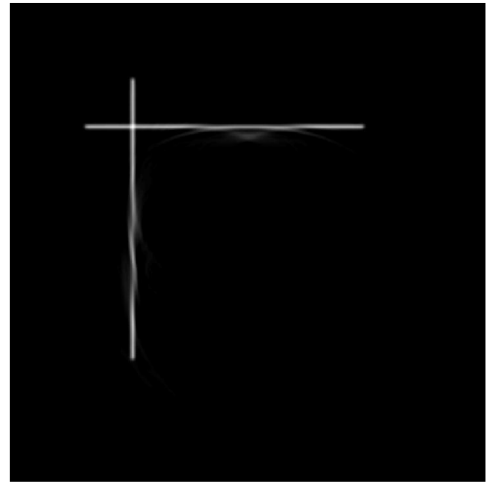

(a)

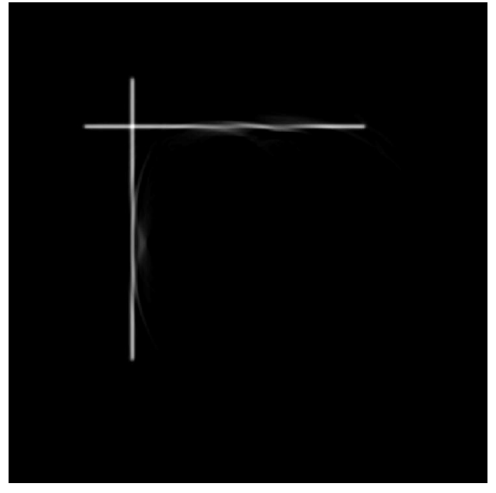

(b)

Figure 3: Images reconstructed using MFISTA where the location of the air void has been shifted $0.2 \mathrm{~mm}$ from its true location in (a) the x-direction and (b) the y-direction. The size and acoustic properties of the air voids otherwise match the true phantom. Images are shown after 40 iterations in a grayscale window of $[0.0,1.0]$.

reconstructed image than that obtained with the true sound speed distribution. This is due to the introduction of numerical errors in the latter case. For this pixel size, the small wavelength components of the pressure wave cannot be accurately modeled as the wave passes through the low sound speed region of the air void.

\section{EXPERIMENTAL RESULTS}

The impact of air voids was also investigated using experimental measurements. Similar to the simulation studies, the experimental results were obtained on a system consisting of a circular array with 512 elements and a radius of $25 \mathrm{~mm}$. The transducers were cylindrically focused and had a central frequency of $5 \mathrm{MHz}$. More information on this system can be found in. ${ }^{13}$ The phantom was cylindrical in shape with a $4 \mathrm{~mm}$ air void located at the center. The cylindrical was composed of $2 \%$ agar by weight. Two strongly absorbing black hairs were placed next to the air void (See Fig. 5). The pressure data was recorded at a sampling rate of $40 \mathrm{MHz}$ for 1300 time steps, corresponding to $32.5 \mu \mathrm{s}$.

The images were reconstructed using MFISTA-TV. ${ }^{3,12}$ The simulation grid consisted of $2048 \times 2048$ pixels with a pixel size of $0.25 \mathrm{~mm}$. The measured pressure data was upsampled to a sampling rate of $120 \mathrm{MHz}$ by use of linear interpolation, and a Hann-window low-pass filter with a cutoff frequency of $6 \mathrm{MHz}$ was applied to each time trace. A step size of $1 / 3$ and a regularization parameter value of $10^{-3}$ were employed. 


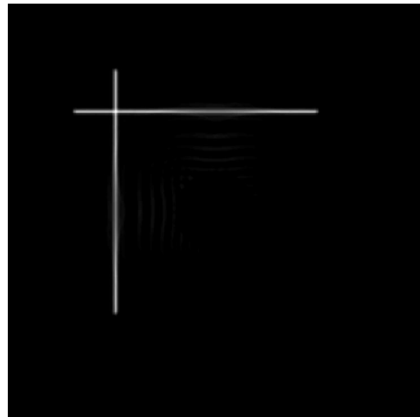

(a)

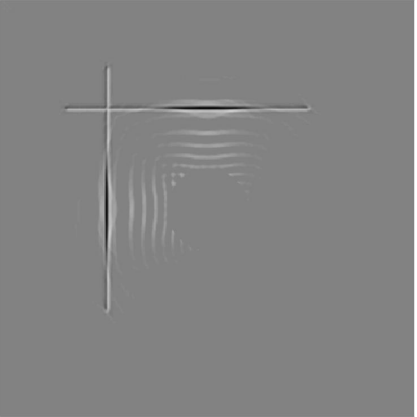

(b)

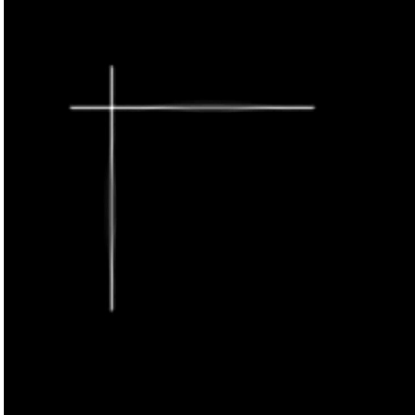

(c)

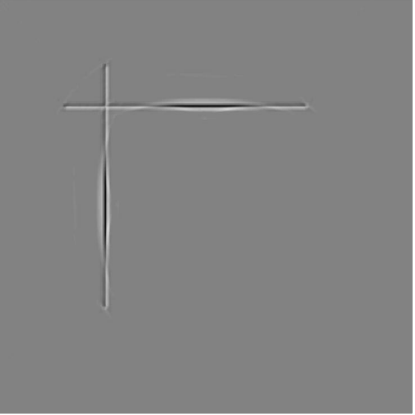

(d)

Figure 4: (a) Reconstructed image and (b) error map for the case where the sound speed and density variations of the medium are included. (c) Reconstructed image and (d) error map for the case where only density variations are included. The reconstructed images are shown after 40 iterations in a grayscale of $[0.0,1.0]$. The error maps are shown in a grayscale of $[-0.2,0.2]$.

While the impact of the air void can obviously be seen, determining its location from the reconstructed image is still challenging. To identify the location of the air void, the air void was segmented from photograph of the phantom. This photograph was scaled to match the true size of the air void. The photograph was then co-registered with the reconstructed image obtained assuming a homogeneous medium using the locations of the two hairs. The location of the air void was further adjusted to correct for the perspective of the photograph. From this, an acoustic model for the medium was developed assuming typical values for the sound speed and density of air and water. The acoustic properties of the agar were not treated separately from the larger water bath.

Images were reconstructed assuming an acoustically homogeneous medium and the heterogeneous medium obtained by the procedure above (See Fig. 5). When a homogeneous medium is employed, the reconstructed image contains significant artifacts, similar in nature to those seen in the computer simulation studies. These artifacts are eliminated when the estimated acoustic properties of the air void are included in the model.

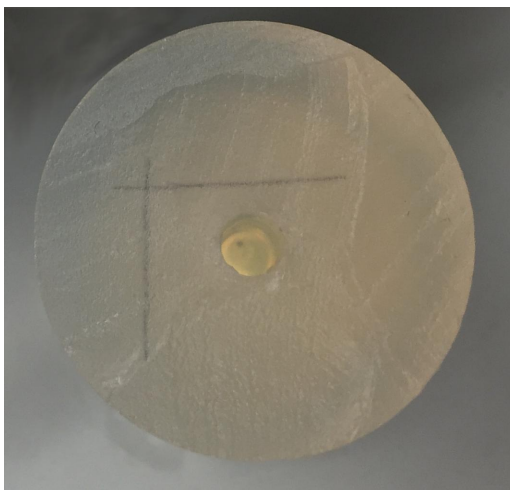

(a)

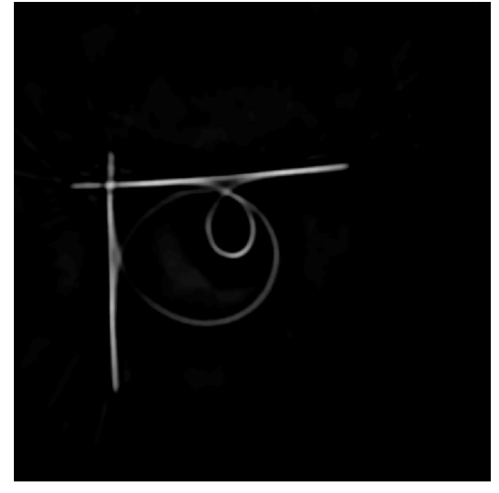

(b)

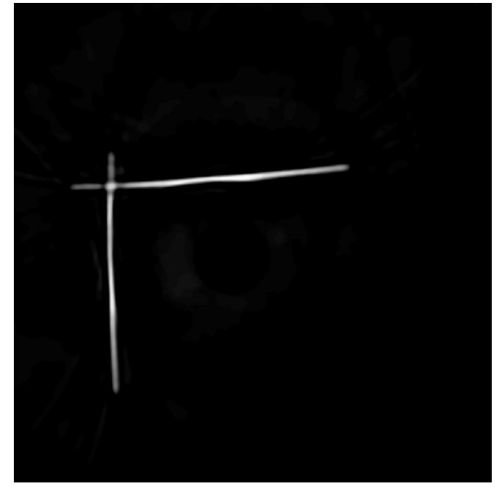

(c)

Figure 5: (a) A photograph of the experimental phantom. Images reconstructed assuming (b) a homogeneous medium and (c) the estimated acoustic properties of the air void. The reconstructed images are shown in a grayscale window of $[0.00,0.25]$.

\section{SUMMARY}

The presence of air voids can lead to significant artifacts in reconstructed images if the medium is assumed to be acoustically homogeneous. Image reconstruction methods based on the acoustic wave equation that explicitly 
model sound speed and density variations can largely eliminate these artifacts. However, accurate knowledge of the location and size of the air voids is needed. The low sound speed of air can result in strict requirements on the pixel size and time step for the numerical wave solver. For air voids, this computational burden can be reduced by ignoring sound speed variations and accounting only for density. This approach can produce more accurate reconstructed images than obtained by use of the true medium when the pixel size is large. This benefit may be more fully realized in 3D image reconstruction problems. Co-registration with an adjunct imaging modality can also aid in the identification of air void locations. Here, a photograph was employed, but information from other modalities could be substituted.

\section{ACKNOWLEDGMENTS}

This work was supported in part by NIH awards CA1744601 and EB01696301.

\section{REFERENCES}

[1] Wang, L. V., "Multiscale photoacoustic microscopy and computed tomography," Nature Photonics 3, 503509 (Sept. 2009).

[2] Wang, L. V. and Hu, S., "Photoacoustic Tomography: In Vivo Imaging from Organelles to Organs," Science 335, 1458-1462 (Mar. 2012).

[3] Huang, C., Wang, K., Nie, L., Wang, L., and Anastasio, M., "Full-Wave Iterative Image Reconstruction in Photoacoustic Tomography With Acoustically Inhomogeneous Media," IEEE Transactions on Medical Imaging 32, 1097-1110 (June 2013).

[4] Xu, Y. and Wang, L. V., "Effects of acoustic heterogeneity in breast thermoacoustic tomography," IEEE Transactions on Ultrasonics, Ferroelectrics and Frequency Control 50(9), 1134-1146 (2003).

[5] Jose, J., Willemink, R. G. H., Steenbergen, W., Slump, C. H., Leeuwen, T. G. v., and Manohar, S., "Speedof-sound compensated photoacoustic tomography for accurate imaging," Medical Physics 39, 7262-7271 (Dec. 2012).

[6] Jiang, H., Yuan, Z., and $\mathrm{Gu}, \mathrm{X}$., "Spatially varying optical and acoustic property reconstruction using finite-element-based photoacoustic tomography," JOSA A 23(4), 878-888 (2006).

[7] Tabei, M., Mast, T. D., and Waag, R. C., "A k-space method for coupled first-order acoustic propagation equations," The Journal of the Acoustical Society of America 111(1), 53-63 (2002).

[8] Cox, B. T., Kara, S., Arridge, S. R., and Beard, P. C., "k-space propagation models for acoustically heterogeneous media: Application to biomedical photoacoustics," The Journal of the Acoustical Society of America 121(6), 3453 (2007).

[9] Mast, T. D., Souriau, L. P., Liu, D.-L., Tabei, M., Nachman, A. I., and Waag, R. C., "A k-space method for large-scale models of wave propagation in tissue," IEEE Transactions on Ultrasonics, Ferroelectrics and Frequency Control 48(2), 341-354 (2001).

[10] Prince, J. L. and Links, J. M., [Medical Imaging: Signals and Systems], Pearson Prentice Hall, Upper Saddle River, NJ (2006).

[11] Treeby, B. and Cox, B., "k-Wave: MATLAB toolbox for the simulation and reconstruction of photoacoustic wave-fields," Journal of Biomedical Optics 15(2), 021314 (2010).

[12] Beck, A. and Teboulle, M., "Fast gradient-based algorithms for constrained total variation image denoising and deblurring problems," IEEE Transactions on Image Processing 18(11), 2419-2434 (2009).

[13] Xia, J., Chatni, M. R., Maslov, K., Guo, Z., Wang, K., Anastasio, M., and Wang, L. V., "Whole-body ring-shaped confocal photoacoustic computed tomography of small animals in vivo," Journal of biomedical optics 17(5), 0505061-0505063 (2012). 